\title{
Genome-Wide Linkage Mapping Reveals Stripe Rust Resistance in Common Wheat (Triticum aestivum) Xinong1376
}

\author{
Jingmei Mu, ${ }_{1}^{1}$ Jianhui Wu, ${ }^{1}$ Shengjie Liu, ${ }^{1}$ Miaofei Dai, ${ }^{1}$ Daojie Sun, ${ }^{1}$ Shuo Huang, ${ }^{1}$ Qilin Wang, ${ }^{1}$ Qingdong Zeng, ${ }^{1}$ Shizhou Yu, ${ }^{1}$ \\ Li Chen, ${ }^{2}$ Zhensheng Kang, ${ }^{1, \dagger}$ and Dejun Han ${ }^{1, \dagger}$ \\ ${ }^{1}$ State Key Laboratory of Crop Stress Biology for Arid Areas, Northwest A\&F University, Yangling, 712100, Shaanxi, China \\ ${ }^{2}$ Extension Center for Agriculture Technology, Agriculture Department of Tibetan Autonomous Region, China
}

\begin{abstract}
Stripe rust, also known as yellow rust, is a significant threat to wheat yield worldwide. Adult plant resistance (APR) is the preferred way to obtain durable protection. Chinese winter wheat cultivar Xinong 1376 has maintained acceptable APR to stripe rust in field environments. To characterize APR in this cultivar, $190 \mathrm{~F}_{10}$ recombinant inbred lines (RILs) developed from Xiaoyan $81 \times$ Xinong 1376 were evaluated for infection type and disease severity in fields either artificially or naturally inoculated. The population along with parents were genotyped using the Illumina $90 \mathrm{~K}$ singlenucleotide polymorphism arrays. Six quantitative trait loci (QTL) were detected using the inclusive composite interval mapping method. QYr.nwafu- $4 A L$ and

likely corresponded to a gene-rich region on the long arm of chromosomes 4A and 6B. QYr.nwafu-5AL, QYr.nwafu-5BL, QYr.nwafu$3 B L .1$, and $Q Y$ r.nwafu-3BL.2 were detected only in some environments but enhanced the level of resistance conferred by QYr.nwafu- $4 A L$ and QYr.nwafu-6BL.3. Kompetitive allele-specific PCR (KASP) markers developed for $Q Y r$ r.nwafu- $4 A L$ and $Q Y$ r.nwafu-6BL.3 were confirmed in a subset of RILs and 133 wheat genotypes. The QTL on $4 A L$ and 6BL with their linked KASP markers would be useful for marker-assisted selection to improve stripe rust resistance in breeding programs.
\end{abstract} QYr.nwafu-6BL.3 conferred stable resistance in all environments, and
Keywords: cereals and grains, disease management, field crops, fungi
Wheat is grown on 215 million ha each year (CCAFS 2017). As the staple food of humans, the demand for wheat is expected to rise with increases in the population (Ray et al. 2012). Stripe rust, caused by Puccinia striiformis $\mathrm{f}$. tritici, has become a significant threat to grain yield and quality in most wheat-growing regions, and the crop losses from epidemics range from 5 to $25 \%$, sometimes $100 \%$ on highly susceptible wheat cultivars (Chen 2014; Wellings 2011). Although chemical control is currently a major approach to reduce yield losses, the cost and potential environmental effects are a global concern (Chen 2014). Over the long term, stripe rust management should rely on growing resistant cultivars (Chen 2005; McIntosh et al. 1995).

Rust resistance is broadly but not exclusively grouped into seedling stage and adult plant categories. Compared with seedling resistance, adult plant resistance (APR) becomes increasingly effective with age, and more likely to be durable (Chen 2013). To date, more than eighty $\mathrm{Yr}$ genes have been identified and formally named (McIntosh et al. 2017). Most of them had seedling resistance that has been defeated by predominant virulent races. More than 200 quantitative trait loci (QTL) have also been reported (Bulli et al. 2016; Maccaferri et al. 2015; Zeng et al. 2019a). Because these QTL provided

${ }^{\dagger}$ Corresponding authors: Z. Kang; kangzs@ nwsuaf.edu.cn; and D. Han; handj@nwsuaf.edu.cn

J. Mu, J. Wu, and S. Liu contributed equally to this work.

Funding: This study was financially supported by the National key "SciTech" project in China (2016ZX08002001), the National Key Research and Development Program of China (number 2016YFD0101802), the earmarked fund for Modern Agro-industry Technology Research System (number CARS-3-1-11), and the key project Science and Technology of Tibetan Autonomous Region, China (number XZ201702NB15).

*The $\boldsymbol{e}$-Xtra logo stands for "electronic extra" and indicates that two supplementary tables are published online.

The author(s) declare no conflict of interest.

Accepted for publication 10 June 2019.

() 2019 The American Phytopathological Society partial resistance and were easily affected by environmental conditions, cultivars with APR do succumb to yield losses in epidemic situations (Ellis et al. 2014). Therefore, identification of more APR genes to combine with effective all-stage resistance (ASR) genes is important to increase the level of protection and durability of resistance.

Although pyramiding resistance genes for high levels of resistance can be achieved using traditional breeding and phenotypic selection, breeding programs increasingly rely on molecular marker-based selection. Single-nucleotide polymorphism (SNP) markers arrayed in different platforms such as the $9 \mathrm{~K}$ and $90 \mathrm{~K}$ chips from Illumina (Cavanagh et al. 2013; Wang et al. 2014) and 660K and 820K chips from Affymetrix (Cui et al. 2017; Winfield et al. 2016), with many advantages over earlier marker types in terms of throughput, cost, and specificity, are now available for crop genetic studies and plant breeding programs (Allen et al. 2011; Colasuonno et al. 2014; Jia and Zhao 2016; Mu et al. 2019; Wu et al. 2017). Genome-wide linkage mapping based on high-throughput SNP arrays provides a rapid approach to find markers linked to specific genes (Wu et al. 2018a; Zeng et al. 2019b).

Wheat cultivar Xinong1376 (pedigree: 84G/Bi16) with 1BL/1RS translocation developed by Northwest A\&F University in Shaanxi province has been widely used as the major donor parent in breeding programs due to its good agronomic traits and high grain yield (Wang 1996). Xinong1376 has been included in stripe rust nurseries in Shaanxi and Gansu since 2008 and consistently showed a high level of APR (Han et al. 2012). However, the genetic basis of this resistance remains unknown. Therefore, the objectives of this study were to (i) characterize and dissect the genetic basis of wheat cultivar Xinong1376, (ii) identify QTL for stripe rust APR in an Xiaoyan81 $\times$ Xinong1376 RIL population across multiple environments using SNP markers, and (iii) validate the value of linked Kompetitive allele-specific PCR (KASP)-SNP markers with major QTL for marker-assisted breeding.

\section{Materials and Methods}

Plant materials. Xinong 1376 was crossed as male parent with Xiaoyan81, a major commercial cultivar that is moderately susceptible in the field. In all, $190 \mathrm{~F}_{10}$ recombinant inbred lines (RILs) and the parental lines were assessed for stripe rust response in field 
experiments and genotyped with the 90K SNP array. In total, 133 wheat cultivars and breeding lines from the Yellow and Huai River Valley wheat zone were used to evaluate the polymorphism of markers flanking the major resistance QTL in Xinong1376. Xiaoyan 22 (XY22), Avocet S (AvS), and Mingxian169 (MX169) were planted as susceptible checks throughout this study.

Greenhouse test. Seedling and adult plant tests were conducted under controlled greenhouse conditions to characterize stripe rust resistance in Xinong 1376 and Xiaoyan81. CYR34, the most prominent race in China, was used. The avirulence-virulence pattern of this race has previously been reported (Liu et al. 2017; Wu et al. 2016). For the seedling test, approximately 15 seeds were grown in 9-by-9-by-9-cm pots and, for adult plant tests, three plants were grown in large pots $(20 \mathrm{~cm}$ in diameter by $15 \mathrm{~cm}$ in height $)$ filled with soil mixture. Each pot was placed in a growth chamber at a diurnal temperature cycle, gradually changing from $4^{\circ} \mathrm{C}$ at 2 A.M. to $20^{\circ} \mathrm{C}$ at 2 P.M. with a 16 -h photoperiod, and plants were watered as needed. Seedlings at the two-leaf stage (14 days after sowing) and adult plants at the boot stage were separately inoculated with urediniospores of each race mixed with talc. Briefly, fresh rust spores were evenly mixed with talc powder at an approximate ratio of 1:20 (urediniospores/talc) in a dry $10-\mathrm{ml}$ plastic tube and the spore/talc mixture was spread on the wheat leaves with a cotton swab. Inoculated plants were incubated in a dew chamber at $10^{\circ} \mathrm{C}$ for $24 \mathrm{~h}$ in the dark, and then transferred to a greenhouse at $17 \pm 2^{\circ} \mathrm{C}$ with a photoperiod of $16 \mathrm{~h}$ of light $(10,000 \mathrm{lux})$ and $8 \mathrm{~h}$ of darkness. Infection type (IT) on the second leaf was recorded when the susceptible check (MX169) showed full sporulation (approximately 15 days after inoculation) using a 0-to-9 scale (Line and Qayoum 1992), where IT $0=$ no visible signs or symptom, IT $1=$ only tiny necrotic or chlorotic flecks, IT $2=$ necrotic or chlorotic blotches (or stripes for adult plants) without any sporulation, IT $3=$ necrotic or chlorotic blotches (or stripes) with trace sporulation, IT $4=$ necrotic or chlorotic blotches (stripes) with light sporulation, IT $5=$ necrotic or chlorotic blotches (stripes) with intermediate sporulation, IT $6=$ necrotic or chlorotic blotches (stripes) with moderate sporulation, IT $7=$ abundant sporulation with necrotic or chlorotic blotches (stripes), IT $8=$ abundant sporulation with chlorosis, and IT $9=$ abundant sporulation without necrosis or chlorosis. Plants with ITs 0 to 3 were considered highly resistant, 4 to 6 were moderately resistant, 7 were moderately susceptible, and 8 to 9 was highly susceptible. ITs were recorded once every 3 days and at least two times, and the highest ITs were used for analysis.

Field test. The $190 \mathrm{~F}_{10}$ RIL population and their parental lines were tested for APR at three experimental sites, including Yangling, Tianshui, and Jiangyou, during the 2016-17 and 2017-18 cropping seasons. All field trials were conducted as a randomized complete block design with two replicates at each location. Each line was planted in a single $1-\mathrm{m}$ row with $25-\mathrm{cm}$ spacing between rows. Each row was sown with approximately 20 seeds of each progeny line or parent. Cultivar XY22 was planted every 20 rows as a susceptible check. The susceptible MX169 and AvS were also grown in the spreader rows around the nursery to increase the inoculum load. Fields in Yangling were artificially inoculated with a mixture of equal quantities of urediniospores of $P$. striiformis $\mathrm{f}$. tritici races CYR32, CYR33, and CYR34 at flag leaf emergence. Briefly, a spore suspension was created in a 1:300 ratio of urediniospores/liquid paraffin and sprayed onto MX169 plants using an electric atomizer (3WBS-16 airbrush; Henan Tianweishi Mechanical Co., Ltd.) after sunset when the moisture for infection was favorable. After inoculation, MX169 plants were completely covered with dark plastic bags to keep moisture for $24 \mathrm{~h}$. The fields in Tianshui and Jiangyou were naturally infected because these two locations are considered hotspots for stripe rust, with ideal conditions for the natural occurrence of this disease. IT and disease severity (DS) were scored for each row when the susceptible checks MX169 and AvS had DS value of $80 \%$ or more. IT data were recorded for each line based on the 0-to-9 scale as described above. Disease severities were assessed visually using percentage of diseased leaf area based on the modified Cobb scale
(Peterson et al. 1948). The scales were recorded with 12 degrees $(1,5,10,20,30,40,50,60,70,80,90$, and $100 \%)$; those read as $100 \%$ represented $37 \%$ of the actual rust-covered surface because the development and destructiveness of the underlying mycelium are almost at a maximum when pustules cover $37 \%$ of the surface. Thus, the recorded 12 degrees of rustiness corresponded to the actual percentage $(0.37,1.83,3.7,7.4,11.1,14.8,18.5,22.2,25.9,29.6$, 33.3 , and $37.0 \%$, respectively) covered by rust pustules.

Analysis of phenotypic data. Mean IT and DS of $F_{10}$ RILs were used to examine the variance within individual environments. Analyses of variance (ANOVA) and Pearson's correlation coefficients were performed using the "AOV" tool in the QTL IciMapping V 4.1 software package (Meng et al. 2015; Wang 2009). Broad-sense heritability was calculated as $h 2 b=\sigma 2 g /(\sigma 2 g+\sigma 2 g e / e+\sigma 2 \varepsilon / r e)$, where $\sigma 2 g$ is $\left(M S_{\mathrm{f}}-M S_{\mathrm{fe}}\right) / r e, \sigma 2 g e$ is $\left(M S_{\mathrm{fe}}-M S_{\mathrm{e}}\right) / r$, and $\sigma 2 \varepsilon$ is $M S_{\mathrm{e}} ; \sigma 2 g=$ genetic variance, $\sigma 2 g e=$ genotype-environment interaction variance, $\sigma 2 \varepsilon=$ error variance, $M S_{\mathrm{f}}=$ mean square of genotypes, $M S_{\mathrm{fe}}=$ mean square of genotype-environment interaction, $M S_{\mathrm{e}}=$ mean square of error, $r=$ number of replications, and $e=$ number of environments.

Molecular genotyping. Genomic DNA of parental lines and $\mathrm{F}_{10}$ RILs were extracted from fresh leaves at the jointing stage using the sodium dodecyl sulfonate method (Weining et al. 1994). DNA was dissolved in sterile deionized distilled (dd) $\mathrm{H}_{2} \mathrm{O}$ as stock solutions. DNA concentrations were determined using both $1.0 \%$ agarose gel electrophoresis and the NanoDrop 2000 spectrophotometer (Thermo Fisher Scientific). DNA stocks were diluted to $50 \mathrm{ng} / \mu \mathrm{l}$ with $\mathrm{ddH}_{2} \mathrm{O}$ for use as templates. All 190 RILs and the parental lines were genotyped using the Illumina 90K SNP chip from CapitalBio Corporation (http://www.capitalbio.com). SNP genotype calling and clustering was processed using Genome Studio v1.0 software (Illumina). SNP filtering criteria were followed: monomorphic SNPs and those showing call rates less than $85 \%$, ambiguity in calling, missing rate above $10 \%$, and minor allele frequencies below $5 \%$ were excluded from the dataset. Polymorphic SNPs between parents contained homozygous and heterozygous genotypes. Only homozygous genotype differences were assigned to chromosomes according to $90 \mathrm{~K}$ wheat consensus maps (Wang et al. 2014; Wu et al. 2018a).

Genetic linkage map. SNP markers were tested using a $\chi^{2}$ test to filter markers with significantly distorted segregation before constructing the genetic linkage maps. Genetic linkage map creation and QTL mapping were performed using IciMapping V4.1 software (http://www.isbreeding.net). Redundant markers with the same segregation were merged together using the "BIN" function (Meng et al. 2015). The remaining markers were used to construct the genetic map using the "MAP" function, and the logarithm of odds (LOD) thresholds were set at the range of 6 to 10 . The criteria followed marker grouping, ordering, and ripping, with the nnTwoOpt algorithm and SARF criterion, respectively. The genetic map was drawn using Mapchart v2.3 (Voorrips 2002). The chromosome locations of linkage groups were determined according to the $90 \mathrm{~K}$ wheat consensus map.

QTL mapping. The detection of QTL for IT and DS were based on the BIP function in IciMapping V 4.1, using the inclusive composite interval mapping (ICIM) method. QTL comparisons with previous resistance genes or QTL were made based on two integrated genetic maps. The first consisted of simple-sequence repeat (SSR) markers and the Wheat90K SNP array (Maccaferri et al. 2015), and the second contained known PCR markers (SSR, expressed sequence tag, sequence-tagged site, random amplified polymorphic DNA, and restriction fragment length polymorphism), diversity arrays technology, and SNPs from Wheat $90 \mathrm{~K}$, Wheat $660 \mathrm{~K}$, and Wheat820K SNP arrays (F. Cui, personal communication). All genes or QTL were placed in an integrated genetic map based on the locations of flanking markers. The physical locations of flanked markers were determined through a BLAST search (IWGSC RefSeq v.1.0). The BLAST hits criteria included an e-value threshold of $10^{-5}$ and minimum similarity cutoff greater than $95 \%$ between query and database sequences. 
Conversion of SNP to KASP markers. SNP markers flanking stable QTL were converted to KASP markers for use in breeding programs. KASP markers were developed using a similar approach described in Ramirez-Gonzalez et al. (2015) and Wu et al. (2017, 2018c). Briefly, the sequences of the flanking markers were uploaded to PolyMarker (http://www.polymarker.info/), and only chromosomespecific KASP markers were synthesized. KASP assays were conducted in a 384-well plate format following the protocol of LGC Genomics. Reaction mixtures consisted of final volumes of $5 \mu \mathrm{l}$ containing $2.5 \mu \mathrm{l}$ of genomic DNA (50 to $100 \mathrm{ng}$ ), $2.5 \mu \mathrm{l}$ of $2 \times$ KASP master mix (V4.0; LGC Genomics), and $0.014 \mu \mathrm{l}$ of primer mix (12 $\mathrm{mM}$ each allele-specific primer and $30 \mathrm{mM}$ common primer). A Veriti 384-well thermal cycler (Applied Biosystems) was used with cycling conditions of denaturation at $95^{\circ} \mathrm{C}$ for $15 \mathrm{~min}$, nine cycles of $95^{\circ} \mathrm{C}$ for $20 \mathrm{~s}$, touchdown starting at $65^{\circ} \mathrm{C}$ for $60 \mathrm{~s}$ (decreasing $0.8^{\circ} \mathrm{C}$ per cycle), finally followed by 30 to 40 cycles of amplification $\left(95^{\circ} \mathrm{C}\right.$ for $20 \mathrm{~s}$ and $57^{\circ} \mathrm{C}$ for $60 \mathrm{~s}$ ). End-point fluorescence data were visualized with a microplate reader (FLUOstar Omega; BMG LABTECH) and analyzed using Klustering Caller software (LGC).

\section{Results}

Greenhouse test. Xiaoyan81 along with the susceptible checks MX169 and AvS were susceptible (IT 7 to 9) to CYR34 either seedlings or adults. While Xinong 1376 was susceptible (IT 8 to 9) to CYR34 in seedling test, but highly resistant (IT 1 to 2) to CYR34 in adult-plant stage test.

Field test. The response of 190 RILs and parental lines at the adult plant stage displayed significant variation in all environments. Xinong1376 exhibited a high level of resistance (IT 1, DS $\leq$ $10 \%$ ), and that of Xiaoyan81 was susceptible (IT 7, DS $\geq 70 \%$ ). Both IT and DS data for the RILs showed a normal distribution in all field tests, suggesting that the APR was quantitatively inherited (Fig. 1). ANOVA showed significant phenotypic variation for both IT and DS between different lines, environments, and line-environment
A

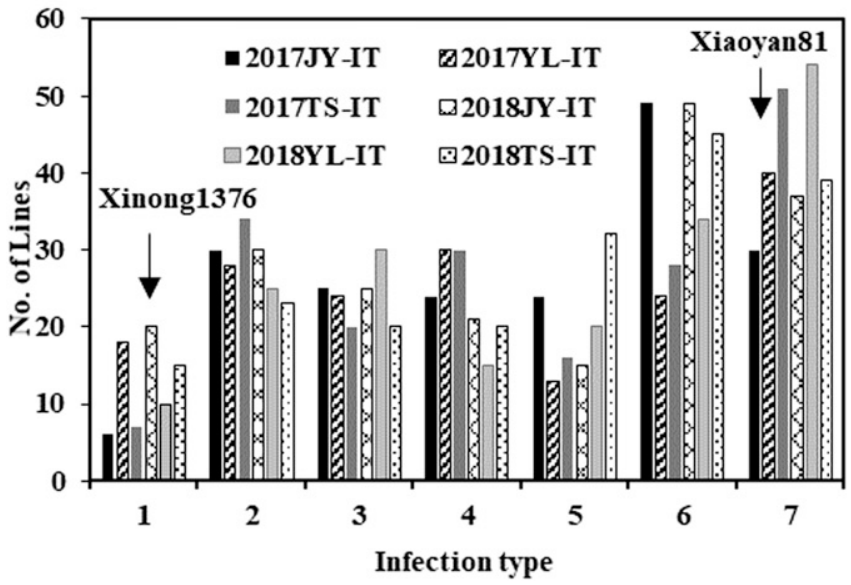

B

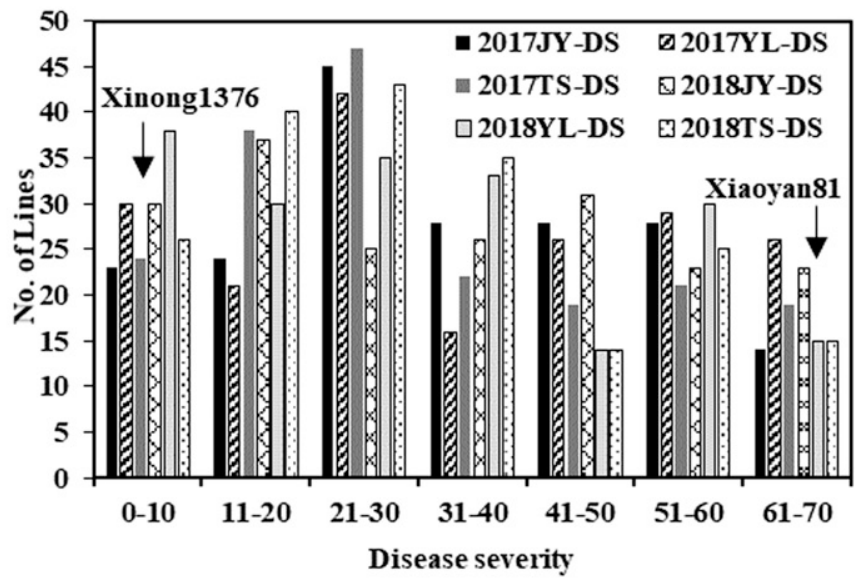

Fig. 1. Frequency distributions of Xiaoyan81 $\times$ Xinong1376 recombinant inbred lines for stripe rust A, infection type (IT) and B, disease severity (DS) in field trials at Jiangyou in 2016-2017 (2017JY) and 2017-18 (2018JY), Yangling in 2016-17 (2017YL) and 2017-18 (2018YL), and Tianshui in 2016-17 (2017TS) and 2017-18 (2018TS). Values for the parents Xiaoyan81 and Xinong1376 are indicated by arrows.

Table 1. Analysis of variance and estimate of broad-sense heritability of stripe rust infection type (IT) and disease severity (DS) among Xiaoyan81 × Xinong 1376 recombinant inbred lines tested at Yangling, Tianshui, and Jiangyou in 2017 and 2018

\begin{tabular}{|c|c|c|c|c|c|c|c|c|}
\hline \multirow[b]{2}{*}{ Source of variation } & \multicolumn{4}{|c|}{ IT } & \multicolumn{4}{|c|}{ DS } \\
\hline & df & Mean square & $F$ value & $P$ value & df & Mean square & $F$ value & $P$ value \\
\hline Lines & 189 & 19.96 & 15.33 & $<0.0001$ & 189 & $2,352.87$ & 13.65 & $<0.0001$ \\
\hline Replicates/environment & 6 & 6.14 & 4.71 & $\ldots$ & 6 & $2,309.33$ & 13.39 & $\ldots$ \\
\hline Environments & 5 & 209.38 & 160.76 & $<0.0001$ & 5 & $19,322.70$ & 112.05 & $<0.0001$ \\
\hline Line-Environments & 944 & 3.42 & 2.63 & $<0.0001$ & 944 & 386.25 & 2.24 & $<0.0001$ \\
\hline Error & 1,101 & 1.30 & $\ldots$ & $\ldots$ & 1,101 & 172.44 & $\ldots$ & $\ldots$ \\
\hline$h 2 b$ & 0.85 & $\ldots$ & $\ldots$ & $\ldots$ & 0.85 & $\ldots$ & $\ldots$ & $\ldots$ \\
\hline
\end{tabular}

Table 2. Correlation coefficients $(r)$ of infection type (IT) and disease severity (DS) of Xiaoyan $81 \times$ Xinong 1376 recombinant inbred lines (RILs) across six environments ${ }^{\mathrm{a}}$

\begin{tabular}{|c|c|c|c|c|c|c|}
\hline \multirow[b]{2}{*}{ Environment } & \multicolumn{6}{|c|}{$r$ value based on DS (IT) ${ }^{b}$} \\
\hline & 2017JY & 2017YL & 2017TS & 2018JY & 2018YL & 2018TS \\
\hline $2017 \mathrm{JY}$ & 1 & $\ldots$ & $\ldots$ & $\ldots$ & $\ldots$ & $\ldots$ \\
\hline 2017YL & $0.84(0.76)$ & 1 & $\ldots$ & $\ldots$ & $\ldots$ & $\ldots$ \\
\hline 2017TS & $0.75(0.65)$ & $0.76(0.68)$ & 1 & $\ldots$ & $\ldots$ & $\ldots$ \\
\hline 2018JY & $0.85(0.75)$ & $0.80(0.78)$ & $0.65(0.76)$ & 1 & $\ldots$ & $\ldots$ \\
\hline 2018YL & $0.65(0.70)$ & $0.75(0.76)$ & $0.76(0.83)$ & $0.86(0.76)$ & 1 & $\ldots$ \\
\hline 2018TS & $0.70(0.80)$ & $0.72(0.74)$ & $0.74(0.71)$ & $0.81(0.75)$ & $0.73(0.71)$ & 1 \\
\hline
\end{tabular}

a Year and location: 2017 and 2018 represent the RIL populations grown during the 2016-17 and 2017-18 cropping seasons, respectively; and JY, YL, and TS denote Jiangyou, Yangling, and Tianshui, respectively.

${ }^{\mathrm{b}} r$ Values calculated with IT are shown in parentheses. All $r$ values are significant at $P<0.001$. 
Table 3. Summary of quantitative trait loci (QTL) for stripe rust resistance detected in the Xiaoyan $81 \times$ Xinong 1376 recombinant inbred line populations across six environments

\begin{tabular}{|c|c|c|c|c|c|c|c|}
\hline QTL, environments ${ }^{\mathbf{a}}$ & Left marker & Right marker & Genetic position $^{\mathbf{b}}$ & Physical interval $^{c}$ & LOD $^{d}$ & $\mathbf{P V E}^{\mathrm{e}}$ & $\overline{\mathrm{ADD}^{\mathrm{f}}}$ \\
\hline \multicolumn{8}{|l|}{ QYr.nwafu- $4 A L$} \\
\hline 2017JY_IT & IWB6683 & IWB49885 & 137 & $617-622$ & 12.5 & 25.9 & -12.5 \\
\hline 2017YL_IT & IWB7070 & IWB6683 & 131 & 617 & 12.7 & 13.2 & -9.1 \\
\hline 2017TS_IT & IWB6683 & IWB49885 & 137 & $617-622$ & 13.2 & 27.5 & -13.8 \\
\hline 2017JY_DS & IWB6683 & IWB49885 & 137 & $617-622$ & 10.4 & 18.7 & -8.5 \\
\hline 2017YL_DS & IWB7070 & IWB6683 & 131 & 617 & 15.2 & 20.2 & -10.2 \\
\hline 2017TS_DS & IWB6683 & IWB49885 & 137 & $617-622$ & 11.3 & 23.5 & -10.8 \\
\hline 2017_mean & IWB6683 & IWB49885 & 137 & $617-622$ & 13.5 & 24.7 & -11.1 \\
\hline 2018JY_IT & IWB6683 & IWB49885 & 137 & $617-622$ & 18.7 & 30.5 & -17.5 \\
\hline 2018YL_IT & IWB7070 & IWB6683 & 131 & 617 & 8.4 & 25.3 & -9.1 \\
\hline 2018TS_IT & IWB7070 & IWB6683 & 131 & 617 & 15.8 & 28.7 & -16.2 \\
\hline 2018JY_DS & IWB6683 & IWB49885 & 137 & $617-622$ & 14.2 & 32.8 & -19.8 \\
\hline 2018YL_DS & IWB6683 & IWB49885 & 137 & $617-622$ & 10.2 & 20.9 & -9.5 \\
\hline 2018TS_DS & IWB6683 & IWB49885 & 137 & $617-622$ & 9.8 & 18.2 & -8.2 \\
\hline 2018_mean & IWB6683 & IWB49885 & 137 & $617-622$ & 12.8 & 25.5 & -11.2 \\
\hline \multicolumn{8}{|l|}{ QYr.nwafu-6BL.3 } \\
\hline 2017JY_IT & IWB52926 & IWB10268 & 12 & $605-617$ & 7.7 & 20.2 & -9.8 \\
\hline 2017YL_IT & IWB10268 & IWB10041 & 14 & $605-696$ & 6.9 & 26.7 & -11.9 \\
\hline 2017TS_IT & IWB2097 & IWB10268 & 12 & $605-613$ & 12.8 & 23.6 & -10.5 \\
\hline 2017JY_DS & IWB2097 & IWB10268 & 12 & $605-613$ & 8.3 & 17.4 & -12.3 \\
\hline 2017YL_DS & IWB52926 & IWB10268 & 12 & $605-617$ & 10.1 & 25.2 & -15.2 \\
\hline 2017TS_DS & IWB2097 & IWB10268 & 12 & $605-613$ & 8.9 & 15.8 & -9.3 \\
\hline 2017_mean & IWB2097 & IWB10268 & 12 & $605-613$ & 11.5 & 25.5 & -8.5 \\
\hline 2018JY_IT & IWB52926 & IWB10268 & 12 & $605-617$ & 9.8 & 23.4 & -9.2 \\
\hline 2018YL_IT & IWB10268 & IWB1004I & 14 & $605-696$ & 11.2 & 25.6 & -12.9 \\
\hline 2018TS_IT & IWB10268 & IWB10041 & 14 & $605-696$ & 10.6 & 18.8 & -11.1 \\
\hline 2018JY_DS & IWB10268 & IWB1004I & 14 & $605-696$ & 7.5 & 15.6 & -8.2 \\
\hline 2018YL_DS & IWB52926 & IWB10268 & 12 & $605-617$ & 8.3 & 20.1 & -11.4 \\
\hline 2018TS_DS & IWB52926 & IWB10268 & 12 & $605-617$ & 7.9 & 16.4 & -6.9 \\
\hline 2018_mean & IWB2097 & IWB10268 & 12 & $605-613$ & 13.8 & 23.2 & -10.1 \\
\hline \multicolumn{8}{|l|}{ QYr.nwafu-5AL } \\
\hline 2017JY_IT & IWB5776 & IWB63558 & 102 & $463-598$ & 5.4 & 8.2 & -5.9 \\
\hline 2017YL_IT & IWB5776 & IWB63558 & 102 & $463-598$ & 6.3 & 7.1 & -5.1 \\
\hline 2017JY_DS & IWB5776 & IWB63558 & 102 & $463-598$ & 4.7 & 9.3 & -8.4 \\
\hline 2017YL_DS & IWB5776 & IWB63558 & 102 & $463-598$ & 5.8 & 8.5 & -6.1 \\
\hline 2017_mean & IWB5776 & IWB63558 & 102 & $463-598$ & 8.1 & 8.9 & -7.2 \\
\hline 2018JY_IT & IWB5776 & IWB63558 & 102 & $463-598$ & 5.1 & 9.5 & -10.2 \\
\hline 2018YL_IT & IWB5776 & IWB63558 & 102 & $463-598$ & 4.5 & 8.9 & -8.1 \\
\hline 2018JY_DS & IWB5776 & IWB63558 & 102 & $463-598$ & 5.4 & 9.2 & -9.8 \\
\hline 2018YL_DS & IWB5776 & IWB63558 & 102 & $463-598$ & 6.4 & 7.5 & -5.5 \\
\hline 2018_mean & IWB5776 & IWB63558 & 102 & $463-598$ & 6.5 & 9.6 & -9.3 \\
\hline \multicolumn{8}{|l|}{ QYr.nwafu-3B.1 } \\
\hline 2017TS_IT & IWA484I & IWB12466 & 65 & $669-674$ & 4.6 & 9.3 & -4.2 \\
\hline 2017TS_DS & IWA484I & $I W B 12466$ & 65 & $669-674$ & 5.8 & 10.8 & -5.3 \\
\hline 2018TS_IT & IWA484I & IWB12466 & 65 & $669-674$ & 6.5 & 8.3 & -3.1 \\
\hline 2018TS_DS & IWA484I & IWB12466 & 65 & $669-674$ & 5.4 & 9.7 & -5.4 \\
\hline 2017TS_IT & IWA4841 & IWB12466 & 65 & $669-674$ & 5.6 & 9.5 & -3.2 \\
\hline 2017TS_DS & IWA484I & IWB12466 & 65 & $669-674$ & 4.2 & 8.1 & -5.1 \\
\hline 2018TS_IT & IWA484I & IWB12466 & 65 & $669-674$ & 5.4 & 8.5 & -3.9 \\
\hline 2018TS_DS & IWA484I & IWB12466 & 65 & $669-674$ & 4.6 & 9.6 & -5.8 \\
\hline \multicolumn{8}{|l|}{ QYr.nwafu-3B.2 } \\
\hline 2017JY_IT & IWB6460 & IWB32266 & 121 & $590-606$ & 4.5 & 10.6 & 6.6 \\
\hline 2017YL_IT & IWB6460 & IWB32266 & 121 & $590-606$ & 3.7 & 8.9 & 5.4 \\
\hline 2017TS_IT & IWB6460 & IWB32266 & 121 & $590-606$ & 4.1 & 9.1 & 3.2 \\
\hline 2017JY_DS & IWB6460 & IWB32266 & 121 & $590-606$ & 3.5 & 7.2 & 4.8 \\
\hline \multicolumn{8}{|l|}{ QYr.nwafu- $5 B L$} \\
\hline 2017YL_DS & IWB7873 & IWB13284 & 320 & $670-672$ & 3.8 & 8.4 & 5.5 \\
\hline 2017TS_DS & IWB7873 & IWB13284 & 320 & $670-672$ & 4.3 & 9.5 & 4.8 \\
\hline 2017_mean & IWB7873 & IWB13284 & 320 & $670-672$ & 3.9 & 9.1 & 6.3 \\
\hline 2018JY_IT & IWB7873 & IWB13284 & 320 & $670-672$ & 4.4 & 8.2 & 3.6 \\
\hline 2018YL_IT & IWB7873 & IWB13284 & 320 & $670-672$ & 5.4 & 9.7 & 4.2 \\
\hline 2018TS_IT & IWB7873 & IWB13284 & 320 & $670-672$ & 4.8 & 8.2 & 4.9 \\
\hline 2018JY_DS & IWB7873 & IWB13284 & 320 & $670-672$ & 3.6 & 9.1 & 3.9 \\
\hline 2018YL_DS & IWB7873 & IWB13284 & 320 & $670-672$ & 4.3 & 10.1 & 4.1 \\
\hline 2018TS_DS & IWB7873 & IWB13284 & 320 & $670-672$ & 5.3 & 8.7 & 3.4 \\
\hline 2018_mean & IWB7873 & IWB13284 & 320 & $670-672$ & 5.8 & 9.8 & 4.3 \\
\hline
\end{tabular}

a 2017 and 2018 represent the field experiments during the 2016-2017 and 2017-2018 cropping seasons, respectively; JY, YL, and TS denote Jiangyou, Yangling, and Tianshui, respectively; IT and DS denote infection type and disease severity, respectively; and mean denotes the average value at different environments each year.

${ }^{b}$ Peak position in centimorgans (cM) from the first linked marker of the relevant linkage group.

${ }^{\mathrm{c}}$ Physical location in mega base $(\mathrm{Mb})$ from linked markers in wheat genome.

${ }^{\mathrm{d}} \mathrm{LOD}=$ logarithm of odds score.

${ }^{\mathrm{e}} \mathrm{PVE}=$ percentages of the phenotypic variance explained by individual QTL.

${ }^{\mathrm{f}} \mathrm{ADD}=$ additive effect of the resistance allele. Negative signs indicate the resistance allele from Xinong 1376. 
interaction. Broad-sense heritability was both 0.85 for IT and DS, with correlation coefficients between environments of 0.68 to 0.83 for IT and 0.65 to 0.86 for DS $(P<0.001)$ (Tables 1 and 2$)$.

Genetic linkage map. In total, 9,728 SNP markers out of the $90 \mathrm{~K}$ SNP array showed polymorphism between the parental lines. Among these, 119 SNPs with more than $10 \%$ missing datapoints were excluded. The remaining 9,609 SNPs fell into 859 bins. After removing redundant markers, a final 1,948 markers were used to create the linkage map. These markers were assigned in 42 linkage groups with a total length of 3,698 centimorgans (cM). The A, B, and D genomes contained 869 (44.6\%), 905 (46.5\%), and 174 (8.9\%) markers covering lengths of $1,733,1,477$ and $488 \mathrm{cM}$ with a density of $2.0,1.6$, and $2.8 \mathrm{cM} /$ marker, respectively. Chromosomes $3 \mathrm{~A}, 3 \mathrm{~B}, 4 \mathrm{~B}, 5 \mathrm{~A}, 5 \mathrm{~B}, 6 \mathrm{~B}$, and $7 \mathrm{~B}$ each had one linkage group; chromosomes $1 \mathrm{~A}, 2 \mathrm{~A}, 1 \mathrm{~B}, 2 \mathrm{~B}$, 3D, 4A, 4D, 6A, 6D, 7A, and 7D each had two linkage groups; 1D, 2D, and 5D each had three linkage groups; and 1RS/1BL was a
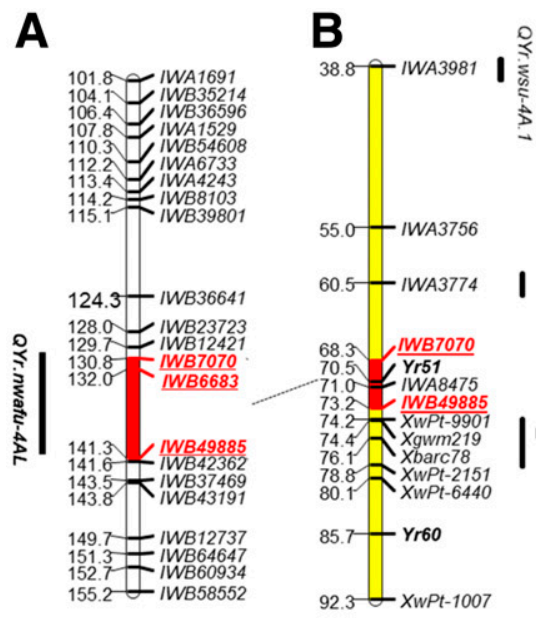

E

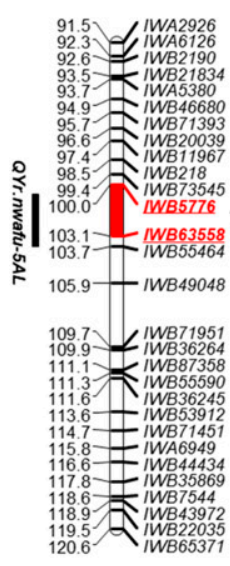

\section{$\mathbf{F}$}

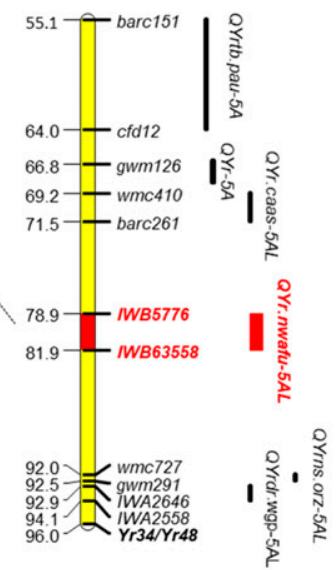

C
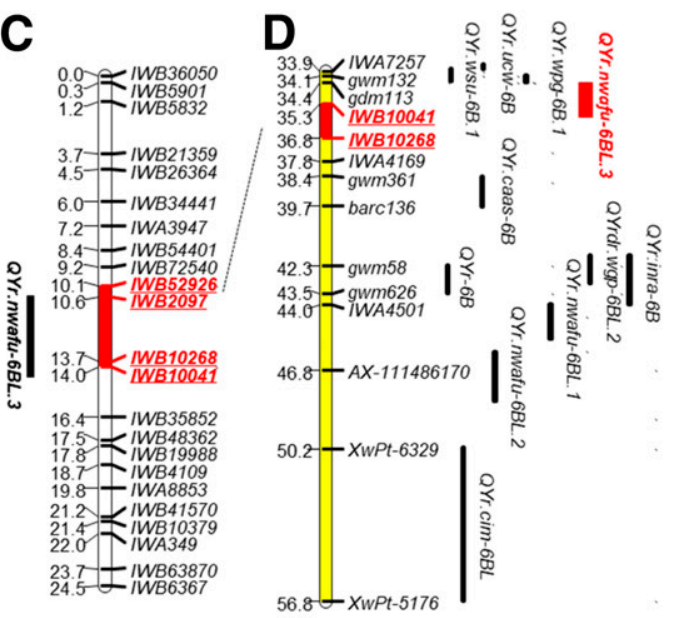

G
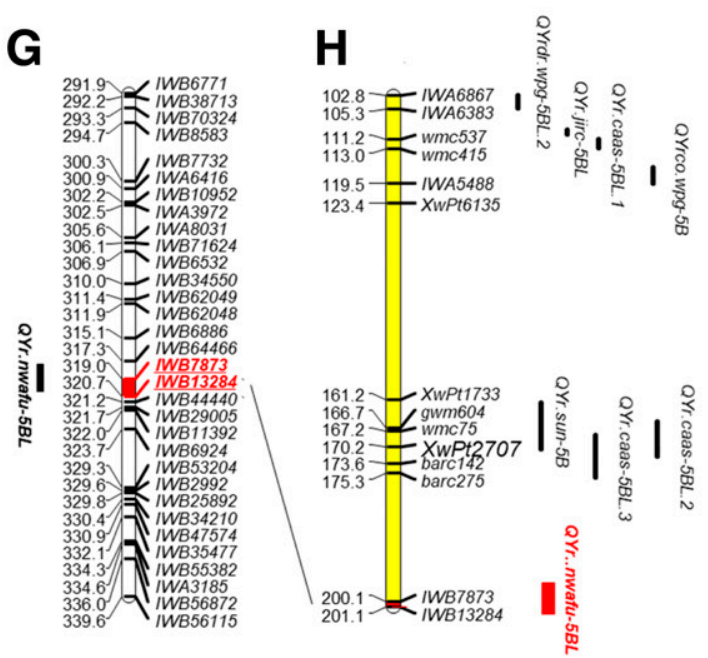

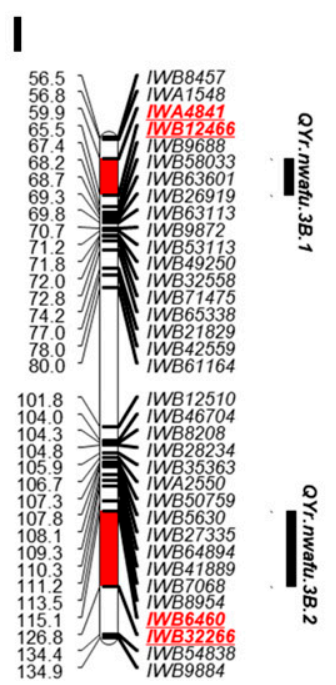

Fig. 2. Genetic maps of recombinant inbred lines from the cross Xiaoyan $81 \times$ Xinong1376. A, C, E, G, and I, Quantitative trait loci (QTL) identified in this study are shown in a linkage map; B, D, F, and H, consensus maps for previously mapped QTL (black bars) were based on integrated genetic maps (Maccaferri et al. 2015) (F. Cui, personal communication). 
translocation line contributed by Xinong1376 that was confirmed in our previous study (Supplementary Table S1). Only linkage groups with significant stripe rust resistance QTL are presented.

QTL mapping. Six QTL were identified on chromosomes 4AL, 6BL, 5AL, 5BL, and 3BL using the ICIM method. Five were from Xinong1376 and QYr.nwafu-5BL was from Xiaoyan81. QYr.nwafu-4AL, QYr.nwafu-6BL.3, QYr.nwafu-5AL, and QYr.nwafu-5BL were detected in almost all six environments; QYr.nwafu-3BL.1 and QYr.nwafu-3BL.2 were each detected in one environment. QYr.nwafu-4AL, with a major effect in reducing stripe rust, was

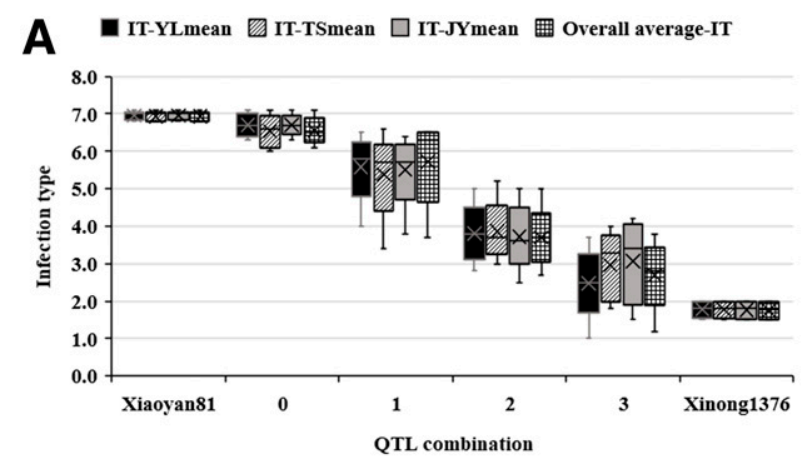

B MDS-YLmean $\square$ MDS-TSmean $\square$ MDS-JYmean Overall average-MDS

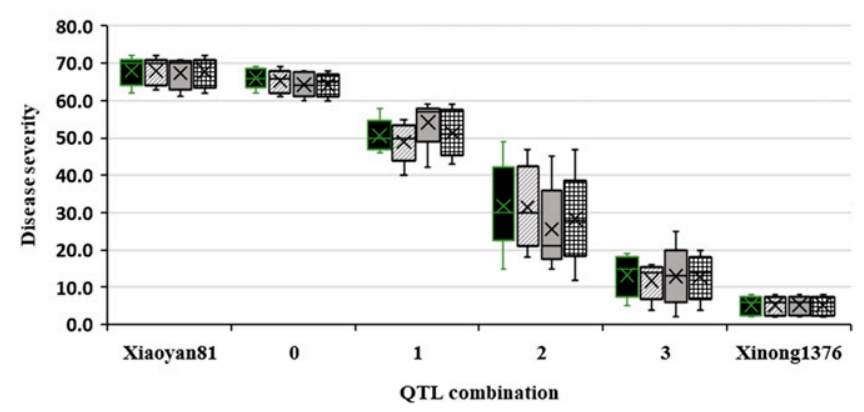

Fig. 3. Effects of quantitative trait loci (QTL) combinations on stripe rust scores in the Xiaoyan81 $\times$ Xinong1376 recombinant inbred line population in Yangling $(\mathrm{YL})$, Tianshui (TS), and Jiangyou (JY) and the mean value (Average) across all environments. Box plots (minima and maxima are black dots, medians are crosses, and the first and third quartiles are boxes) for $\mathbf{A}$, infection type (IT) and B, maximum disease severity (MDS) associated with the identified QTL and their combinations. X-axis refers to number of QTL combinations and Y-axis refers to the value of IT and DS. flanked by SNP markers IWB49885 and IWB7070, explaining 25.3 to $30.5 \%$ of the phenotypic variation in IT and 18.2 to $32.8 \%$ in DS. QYr.nwafu-6BL.3, with the closest markers IWB2097 and IWB10268 spanning $3.1 \mathrm{cM}$, explained 18.8 to $26.7 \%$ of the variation in IT and 15.6 to $25.2 \%$ in DS. QYr.nwafu-5AL flanked by markers IWB5776 and IWB63558, QYr.nwafu-3BL.1 close to IWA4841 and IWB12466, QYr.nwafu-3BL.2 linked with IWB6460 and IWB32666, and QYr.nwafu-5BL in the interval of IWB7873 and $I W B 13284$ explained the phenotype variation below $10 \%$, respectively. The physical locations for these flanked SNP markers were also obtained based on a BLAST search (IWGSC RefSeq v.1.0) (Table 3; Fig. 2).

To show the effects of individual QTL on stripe rust reaction, the 190 lines that carried different allelic combination at the three QTL on $4 \mathrm{~A}, 6 \mathrm{~B}$, and $5 \mathrm{~A}$ were grouped based on the presence of the closest markers. RILs with only one QTL had a level of resistance similar to that of the susceptible parent Xiaoyan81. RILs with combinations $4 \mathrm{AL}+5 \mathrm{AL}, 6 \mathrm{BL}+5 \mathrm{AL}$, and $4 \mathrm{AL}+6 \mathrm{BL}$ showed comparatively lower response than those with only one QTL. Those with three QTL combinations were even more resistant, whereas RILs with no QTL were most susceptible. The mean IT and DS scores of the different combinations are presented in Figure 3. These results clearly showed that the degree of resistance in Xinong1376 depended upon the additive effect of several QTL with variable individual effects.

Conversion of SNP to KASP markers. QYr.nwafu- $4 A L$ and QYr.nwafu-6BL.3, with large effects detected across all test environments, can be useful for marker-assisted selection. SNP markers within the vicinity of the QTL were used for genotyping a panel of 133 Chinese wheat varieties. Among these flanking SNP markers and flanking sequences submitted to PolyMarker, six chromosomespecific SNPs were selected for conversion to KASP markers and to genotype the parents and panel to confirm polymorphisms. KASP markers from SNP markers IWB6683, IWB7070, IWB42362, and $I W B 12421$ were for QYr.nwafu-4AL and KASP markers from IWB52926 and IWB10041 were for QYr.nwafu-6BL.3. Genotyping results are presented in Supplementary Table S2, and the primer sequences are provided in Table 4. Xinong936 and Xinong182 shared at least three marker alleles with $Q Y r . n w a f u-4 A L$. These two accessions showed APR in fields, indicating that they probably had the same QTL. No other genotypes in the panel carried both markers flanking QYr.nwafu-6BL.3, suggesting that this QTL was only unique to Xinong 1376.

\section{Discussion}

Xinong1376 has shown durable APR in field nurseries since its release in 1992. Five QTL were identified in Xinong1376 on

Table 4. Primer sequences of Kompetitive allele-specific PCR (KASP) markers developed from single-nucleotide polymorphism markers closely linked to stripe rust resistance quantitative trait loci (QTL) with major effect

\begin{tabular}{|c|c|c|}
\hline KASP names ${ }^{a}$ & QTL name & Primer sequence $\left(5^{\prime}-3^{\prime}\right)$ \\
\hline IWB6683-4A-A & QYr.nwafu- $4 A L$ & GAAGGTGACCAAGTTCATGCTggcctcggtgatggaGcC \\
\hline IWB6683-4A-B & QYr.nwafu- $4 A L$ & GAAGGTCGGAGTCAACGGATTggcctcggtgatggaGcA \\
\hline IWB6683-4A-C & QYr.nwafu- $4 A L$ & acctGAGTtggaagcgcgG \\
\hline IWB7070-4A-A & QYr.nwafu- $4 A L$ & GAAGGTGACCAAGTTCATGCTacAtTcaGggettcgacaaG \\
\hline IWB7070-4A-B & QYr.nwafu- $4 A L$ & GAAGGTCGGAGTCAACGGATTacAtTcaGggcttcgacaaT \\
\hline IWB7070-4A-C & QYr.nwafu- $4 A L$ & cgtatcgettgaactcctca \\
\hline IWB42362-4A-A & QYr.nwafu- $4 A L$ & GAAGGTGACCAAGTTCATGCTaaagcactgcaggagcgT \\
\hline IWB42362-4A-B & QYr.nwafu- $4 A L$ & GAAGGTCGGAGTCAACGGATTaaagcactgcaggagcgC \\
\hline IWB42362-4A-C & QYr.nwafu- $4 A L$ & tgatcaggttgctagctttattttG \\
\hline IWB12421-4A-A & QYr.nwafu- $4 A L$ & GAAGGTGACCAAGTTCATGCTcggcacaaactacttcatctgT \\
\hline IWB12421-4A-B & QYr.nwafu- $4 A L$ & GAAGGTCGGAGTCAACGGATTcggcacaaactacttcatctgC \\
\hline IWB12421-4A-C & QYr.nwafu- $4 A L$ & gcgatcttcatgccggaT \\
\hline IWB52926-6B-A & QYr.nwafu-6BL.3 & GAAGGTGACCAAGTTCATGCTtggcgaaatttgctccaaaG \\
\hline IWB52926-6B-B & QYr.nwafu-6BL.3 & GAAGGTCGGAGTCAACGGATTtggcgaaatttgctccaaaA \\
\hline IWB52926-6B-C & QYr.nwafu-6BL.3 & tgtactgctagaaacagtttgct \\
\hline IWB10041-6B-A & QYr.nwafu-6BL.3 & GAAGGTGACCAAGTTCATGCTgccagcgtcgtgaccaaC \\
\hline IWB10041-6B-B & QYr.nwafu-6BL.3 & GAAGGTCGGAGTCAACGGATTgecagegtcgtgaccaaT \\
\hline IWB10041-6B-C & QYr.nwafu-6BL.3 & ctcaggggtgtcatcgagtg \\
\hline
\end{tabular}

\footnotetext{
${ }^{\mathrm{a}}$ KASP names: $\mathrm{A}=$ primers with the added FAM adapter, $\mathrm{B}=$ primers with the added HEX adapter, and $\mathrm{C}=$ common primers.
} 
chromosomes 4AL, 6BL, 5AL, and 3BL and named QYr.nwafu-4AL, QYr.nwafu-6BL.3, QYr.nwafu-5AL, QYr.nwafu-3BL.1, and $Q Y r$. nwafu-3BL.2, respectively. QYr.nwafu-4AL and QYr.nwafu- $6 B L .3$ showed significant effects in almost all environments, whereas the other QTL showed minor effects and expressed only in some environments. An additional QTL from Xiaoyan81, QYr.nwafu-5BL, had a major effect in reducing stripe rust response.

QYr.nwafu-4AL was mapped near the distal end of chromosome arm 4AL (Fig. 2A). Thus far, five APR QTL for stripe rust have been reported on the telomere region of 4AL. Most of the QTL were assigned on the bin 4AL4-0.80-1.00 based on an integrated bin map (Qi et al. 2004; Sourdille et al. 2004). These QTL contained QYrst.orr-4AL in Stephens (Vazquez et al. 2012), QYrid.ui-4A in IDO444 (Chen et al. 2012), QYr-4A in Sachem (Singh et al. 2013), QYr.sgi-4A in Kariega (Agenbag et al. 2014), and QYrns.orz-4AL in NSA-980995 (Vazquez et al. 2015), and two QTL from a genome-wide association study, including $Q Y r . u c w-4 A$ (Maccaferri et al. 2015) and $Q Y r . w s u-4 A$ (Bulli et al. 2016). QYr.nwafu-4AL flanked by marker IWB49885 and IWB6683 also fell into the same chromosome bin based on marker bin location (https://wheat.pw.usda.gov/cgi-bin/westsql/map_locus.cgi) (Sourdille et al. 2004). Thus, it indicated that $Q Y$ r.nwafu- $4 A L$ shared a common genomic region with these QTL. Two formally named genes, $\operatorname{Yr} 51$ and $\operatorname{Yr} 60$, were also in the proximal region. $Y r 51$ was close to $Q Y r$.sgi-4A, with a 4.3-cM distance (Randhawa et al. 2013), and Yr60 was approximately $10.1 \mathrm{cM}$ distant from $\operatorname{Yr} 51$ (Herrera-Foessel et al. 2015). However, both genes conferred ASR. Yr51 was from Pakistan wheat landrace AUS27858 and Yr60 was from Mexican common wheat Almop, whereas Xinong1376 was sourced from Chinese landraces (Wang 1996). The different resistance sources and resistance types indicated that $Q Y$ r.nwafu-4AL was different from Yr51 and Yr60. Because $Q Y r . n w a f u-4 A L$ explained $25.5 \%$ of the mean variation against stripe rust, conferring a moderate level of resistance to stripe rust, combining this QTL with additional genes or QTL will achieve high levels of resistance in the field.

Several stripe rust APR QTL on 6BL have been reported (Bulli et al. 2016; Zeng et al. 2019c). To estimate the relationship of QYr.nwafu$6 B L .3$ from other resistance QTL on 6BL, all genes or QTL were placed in an integrated map based on the genetic locations of flanking markers (Bulli et al. 2016; Maccaferri et al. 2015) and physical location based on the Chinese Spring sequence (IWGSC RefSeq v.1.0). $Q Y r-6 B$ from Pavon76 (William et al. 2006), QYr.inra-6B from Renan (Dedryver et al. 2009), QYrdr.wgp-6BL.2 in Druchamp (Hou et al. 2015), and QYr.nwafu-6BL.1 in Friedrichswerther (Wu et al. 2018b) were placed at 42.3 to $43.5 \mathrm{cM}$, a physical region of about 417 to $521 \mathrm{Mb}$. QYr.nwafu-6BL.2 (Zeng et al. 2019c) was in the region of 50.2 to $56.8 \mathrm{cM}$. Three QTL were concentrated in 33.9 to $39.7 \mathrm{cM}$ of genetic distance, including QYr.wsu-6B.1 (Bulli et al. 2016) and QYr.ucw-6B (Maccaferri et al. 2015) in the interval of 33.9 to 35.3 cM (genomic region of about 631 to $720 \mathrm{Mb}$ ) and QYr.wgp-6B.1 from Stephens (Santra et al. 2008) in 34.5 to $35.0 \mathrm{cM}$ (region of $690 \mathrm{Mb}$ ) whereas, in our study, QYr.nwafu-6BL.3 was in an interval of 35.3 to $36.8 \mathrm{cM}(617$ to $622 \mathrm{Mb})$ according to the flanked markers location (Fig. 2B). QYr.nwafu-6BL.3 seems to be in a proximate region with those three QTL. However, they were accurately distinct in the physical location. Thus, the concise location relationships with the three QTL needs to be further confirmed.

QYr.nwafu-5AL was mapped with flanking markers IWB5776 and IWB63558 (Fig. 2C), and the QTL was identified with high LOD scores in all tested environments, except in Tianshui. The QTL on $5 \mathrm{AL}$ identified in previous studies was mainly distributed in two genomic regions. The first region included Yr34 in wheat line WAWHT2046 (AUS22857/Kulin//Blade), Yr48 in a synthetic derivative spring wheat PI610750 (Croci/Aegilops tauschii (synthetic 205)//Kauz) (Qureshi et al. 2018), QYrdr.wgp-5AL in Druchamp (Vilmorin27/N.R.//Yeoman), and QYrns.orz-5AL in NSA-98-0995 (Hou et al. 2015; Vazquez et al. 2015). QYrtb.pau-5A as hightemperature adult-plant (HTAP) resistance in Triticum boeoticum accession Pau5088 was in the second location (Chhuneja et al. 2008). QYr-5A in a hard red spring wheat Opata 85 (Boukhatem et al. 2002) and QYr.caas-5AL in Pingyuan 50 (Lan et al. 2010) were proximal. However, QYr.nwafu-5AL was located in the distinct region according to the integrated map (Maccaferri et al. 2015; Somers et al. 2004). The pedigree also verified their differences, although Pingyuan 50 was an old Chinese wheat landrace, with an origin similar to Xinong1376. However, Pingyuan 50 displayed slow rusting response (DS 30 to $70 \%$ ) in the same field (Wu et al. 2018b). Therefore, QYr.nwafu-5AL seems to be a novel QTL. Additional haplotype analysis is needed for verification.

QYr.nwafu-5BL was mapped with flanking markers $I W B 7873$ and IWB13284. Several QTL were previously located on chromosome 5BL. QYr.jirc-5BL from Oligoculm, QYr.caas-5BL. 1 from Libellula, and QYrco.wpg-5B from Coda (Case et al. 2014; Lu et al. 2009; Suenaga et al. 2003) should be in the same region (105 to $119 \mathrm{cM}, 43.33$ to 50.37\%). QYr.caas-5BL.2 from Libellula and QYr.sun-5B from Janz seem to be located in the same interval (166.7 to $173.6 \mathrm{cM}, 70.2$ to 73.1\%) (Bariana et al. 2010; Lu et al. 2009) (Fig. 2D). The previously identified QTL were at least $34 \mathrm{cM}$ away from QYr.nwafu-5BL (200.1 cM, 84.31\%) (Maccaferri et al. 2015). Because QYr.nwafu$5 B L$ accounted for only 7.2 to $10.6 \%$ of the overall percentages of the phenotypic variance, it would be useful when combined with other genes for resistance.

QYr.nwafu-3BL.1 and QYr.nwafu-3BL.2 were identified in wellseparated regions with more than $20 \mathrm{cM}$ of genetic distance (Fig. 2E). Because both QTL were unstable across environments, they are potentially less useful than other QTL detected in this study. Several QTL have been reported on 3BL. QYrrns.orz-3BL was detected in hard red winter wheat NSA 980995 (Vazquez et al. 2015). QYrex. wgp-3BL was detected in Express and QYrid.ui-3B.2 in IDO444 (Chen et al. 2012; Lin and Chen 2009) as HTAP resistance. Their resistance sources and resistance type seem different from Xinong1376; thus, additional studies are required for useful comparisons with the current results.

KASP markers tightly linked with major QTL QYr.nwafu-4AL and QYr.nwafu-6BL.3 can be used to pyramid these genes with other genes in breeding programs. QYr.nwafu-6BL.3 was mapped at less than $5 \mathrm{cM}$ genetic interval by markers IWB2097 and IWB10268; therefore, they can be used for marker-based selection. We also determined that the flanking markers of the two stable QTL were highly polymorphic among the wheat cultivars and breeding lines, indicating the uniqueness of these markers for the incorporation of these genes into other backgrounds to develop new cultivars through marker-assisted selection.

\section{Acknowledgments}

We thank R. A. McIntosh, Plant Breeding Institute, University of Sydney, for critical review of this manuscript.

\section{Literature Cited}

Agenbag, G. M., Pretorius, Z. A., Boyd, L. A., Bender, C. M., MacCormack, R., and Prins, R. 2014. High-resolution mapping and new marker development for adult plant stripe rust resistance QTL in the wheat cultivar Kariega. Mol. Breed. 34:2005-2020.

Allen, A. M., Barker, G. L., Berry, S. T., Coghill, J. A., Gwilliam, R., Kirby, S., Robinson, P., Brenchley, R. C., D'Amore, R., McKenzie, N., Waite, D., Hall, A., Bevan, M., Hall, N., and Edwards, K. J. 2011. Transcript-specific, single-nucleotide polymorphism discovery and linkage analysis in hexaploid bread wheat (Triticum aestivum L.). Plant Biotechnol. J. 9:1086-1099.

Bariana, H. S., Bansal, U. K., Schmidt, A., Lehmensiek, A., Kaur, J., Miah, H., Howes, N., and McIntyre, C. L. 2010. Molecular mapping of adult plant stripe rust resistance in wheat and identification of pyramided QTL genotypes. Euphytica 176:251-260.

Boukhatem, N., Baret, P. V., Mingeot, D., and Jacquemin, J. M. 2002. Quantitative trait loci for resistance against yellow rust in two wheat-derived recombinant inbred line populations. Theor. Appl. Genet. 104:111-118.

Bulli, P., Zhang, J., Chao, S., Chen, X. M., and Pumphrey, M. 2016. Genetic architecture of resistance to stripe rust in a global winter wheat germplasm collection. G3 (Bethesda) 6:2237-2253.

Case, A. J., Naruoka, Y., Chen, X. M., Garland-Campbell, K. A., Zemetra, R. S., and Carter, A. H. 2014. Mapping stripe rust resistance in a Brundage $\times$ Coda winter wheat recombinant inbred line population. PLoS One 9:e91758.

Cavanagh, C. R., Chao, S., Wang, S., Huang, B. E., Stephen, S., Kiani, S., Forrest, K., Saintenac, C., Brown-Guedira, G. L., Akhunova, A., See, D., Bai, G., Pumphrey, M., Tomar, L., Wong, D., Kong, S., Reynolds, M., Da Silva, 
M. L., Bockelman, H., Talbert, L., Anderson, J. A., Dreisigacker, S., Baenziger, S., Carter, A., Korzun, V., Morrell, P. L., Dubcovsky, J., Morell, M. K., Sorrells, M. E., Hayden, M. J., and Akhunov, E. 2013. Genome-wide comparative diversity uncovers multiple targets of selection for improvement in hexaploid wheat landraces and cultivars. Proc. Nat. Acad. Sci. U.S.A. 110: 8057-8062.

CCAFS. 2017. 2016 Annual Report to CGIAR Consortium. CGIAR Research Program on Climate Change, Agriculture and Food Security (CCAFS), Copenhagen, Denmark.

Chen, J. L., Chu, C., Souza, E. J., Guttieri, M. J., Chen, X. M., Xu, S., Hole, D., and Zemetra, R. 2012. Genome-wide identification of QTL conferring hightemperature adult-plant (HTAP) resistance to stripe rust (Puccinia striiformis f. sp. tritici) in wheat. Mol. Breed. 29:791-800.

Chen, X. M. 2005. Epidemiology and control of stripe rust (Puccinia striiformis $\mathrm{f}$. sp. tritici) on wheat. Can. J. Plant Pathol. 27:314-337.

Chen, X. M. 2013. High-temperature adult-plant resistance, key for sustainable control of stripe rust. Am. J. Plant Sci. 4:608-627.

Chen, X. M. 2014. Integration of cultivar resistance and fungicide application for control of wheat stripe rust. Can. J. Plant Pathol. 36:311-326.

Chhuneja, P., Kaur, S., Garg, T., Ghai, M., Kaur, S., Prashar, M., Bains, N. S., Goel, R. K., Keller, B., Dhaliwal, H. S., and Singh, K. 2008. Mapping of adult plant stripe rust resistance genes in diploid A genome wheat species and their transfer to bread wheat. Theor. Appl. Genet. 116:313-324.

Colasuonno, P., Gadaleta, A., Giancaspro, A., Nigro, D., Giove, S., Incerti, O., Mangini, G., Signorile, A., Simeone, R., and Blanco, A. 2014. Development of a high-density SNP-based linkage map and detection of yellow pigment content QTL in durum wheat. Mol. Breed. 34:1563-1578.

Cui, F., Zhang, N., Fan, X., Zhang, W., Zhao, C., Yang, L., Pan, R., Chen, M., Han, J., Zhao, X., Ji, J., Tong, Y., Zhang, H., Jia, J., Zhao, G., and Li, J. 2017. Utilization of a Wheat660 K SNP array-derived high-density genetic map for high-resolution mapping of a major QTL for kernel number. Sci. Rep. 7: Article 3788.

Dedryver, F., Paillard, S., Mallard, S., Robert, O., Trottet, M., Nègre, S., Verplancke, G., and Jahier, J. 2009. Characterization of genetic components involved in durable resistance to stripe rust in the bread wheat "Renan". Phytopathology 99:968-973.

Ellis, J. G., Lagudah, E. S., Spielmeyer, W., and Dodds, P. N. 2014. The past, present and future of breeding rust resistant wheat. Front. Plant Sci. 5:641.

Han, D. J., Zhang, P. Y., Wang, Q. L., Zeng, Q. D., and Wu, J. H. 2012. Identification and evaluation of resistance to stripe rust in 1980 wheat landraces and abroad germplasm. Sci. Agric. Sin. 45:5013-5023 [In Chinese with English summary].

Herrera-Foessel, S. A., Singh, R. P., Lan, C. X., Huerta-Espino, J., Calvo-Salazar, V., Bansal, U. K., Bariana, H. S., and Lagudah, E. S. 2015. Yr60, a gene conferring moderate resistance to stripe rust in wheat. Plant Dis. 99:508-511.

Hou, L., Chen, X. M., Wang, M. N., See, D. R., Chao, S. M., Bulli, P., and Jing, J. X. 2015. Mapping a large number of QTL for durable resistance to stripe rust in winter wheat Druchamp using SSR and SNP markers. PLoS One 10: e0126794.

Jia, J., and Zhao, G. 2016. Wheat660 SNP array developed by CAAS.

Lan, C. X., Liang, S. S., Zhou, X. C., Zhou, G., Lu, Q. L., Xia, X. C., and He, Z. H. 2010. Identification of genomic regions controlling adult-plant stripe rust resistance in Chinese landrace Pingyuan 50 through bulked segregant analysis. Phytopathology 100:313-318.

Lin, F., and Chen, X. M. 2009. Quantitative trait loci for non-race-specific, hightemperature adult-plant resistance to stripe rust in wheat cultivar Express. Theor. Appl. Genet. 118:631-642.

Line, R. F., and Qayoum, A. 1992. Virulence, aggressiveness, evolution, and distribution of races of Puccinia striiformis (the cause of stripe rust of wheat) in North America 1968-87. Tech. Bull. No. 1788. USDA-ARS, Washington, DC, U.S.A.

Liu, B., Liu, T., Zhang, Z., Jia, Q., Wang, B., Gao, L., Peng, Y., Jin, S., and Chen, W. 2017. Discovery and pathogenicity of CYR34, a new race of Puccinia striiformis f. sp. tritici in China. Acta Phytopathol. Sin. 47:681-687 [In Chinese with English summary].

Lu, Y. M., Lan, C. X., Liang, S. S., Zhou, X. C., Liu, D., Zhou, G., Lu, Q. L., Jing, J. X., Wang, M. N., Xia, X. C., and He, Z. H. 2009. QTL mapping for adultplant resistance to stripe rust in Italian common wheat cultivars Libellula and Strampelli. Theor. Appl. Genet. 119:1349-1359.

Maccaferri, M., Zhang, J., Bulli, P., Abate, Z., Chao, S., Cantu, D., Bossolini, E., Chen, X. M., Pumphrey, M., and Dubcovsky, J. 2015. A genome-wide association study of resistance to stripe rust (Puccinia striiformis f. sp. tritici) in a worldwide collection of hexaploidy spring wheat (Triticum aestivum L.). G3 (Bethesda) 5:449-465.

McIntosh, R. A., Dubcovsky, J., Rogers, W. J., Morris, C., and Xia, X. C. 2017. Catalogue of Gene Symbols for Wheat: 2017 Supplement. https://shigen. nig.ac.jp/wheat/komugi/genes/macgene/supplement2017.pdf

McIntosh, R. A., Wellings, R. A., and Park, R. F. 1995. Pages 20-26 in: Wheat Rusts: An Atlas of Resistance Genes. CSIRO Publications, East Melbourne, VIC, Australia.

Meng, L., Li, H., Zhang, L., and Wang, J. 2015. QTL IciMapping: Integrated software for genetic linkage map construction and quantitative trait locus mapping in biparental populations. Crop J. 3:269-283.
Mu, J., Huang, S., Liu, S., Zeng, Q., Dai, M., Wang, Q., Wu, J., Yu, S., Kang, Z. and Han, D. 2019. Genetic architecture of wheat stripe rust resistance revealed by combining QTL mapping using SNP-based genetic maps and bulked segregant analysis. Theor. Appl. Genet. 132:443-455.

Peterson, R. F., Campbell, A. B., and Hannah, A. E. 1948. A diagrammatic scale for estimating rust intensity on leaves and stems of cereals. Can. J. Res. 26c: 496-500.

Qi, L. L., Echalier, B., Chao, S., Lazo, G. R., Butler, G. E., Anderson, O. D., Akhunov, E. D., Dvořák, J., Linkiewicz, A. M., Ratnasiri, A., Dubcovsky, J., Bermudez-Kandianis, C. E., Greene, R. A., Kantety, R., La Rota, C. M., Munkvold, J. D., Sorrells, S. F., Sorrells, M. E., Dilbirligi, M., Sidhu, D., Erayman, M., Randhawa, H. S., Sandhu, D., Bondareva, S. N., Gill, K. S., Mahmoud, A. A., Ma, X. F., Gustafson, J. P., Conley, E. J., Nduati, V., Gonzalez-Hernandez, J. L., Anderson, J. A., Peng, J. H., Lapitan, N. L., Hossain, K. G., Kalavacharla, V., Kianian, S. F., Pathan, M. S., Zhang, D. S., Nguyen, H. T., Choi, D. W., Fenton, R. D., Close, T. J., McGuire, P. E., Qualset, C. O., and Gill, B. S. 2004. A chromosome bin map of 16,000 expressed sequence tag loci and distribution of genes among the three genomes of polyploid wheat. Genetics 168:701-712.

Qureshi, N., Bariana, H. S., Zhang, P., McIntosh, R. A., Bansal, U. K., Wong, D., Hayden, M. J., Dubcovsky, J., and Shankar, M. 2018. Genetic relationship of stripe rust resistance genes $\mathrm{Yr} 34$ and $\mathrm{Yr} 48$ in wheat and identification of linked KASP markers. Plant Dis. 102:413-420.

Ramirez-Gonzalez, R. H., Uauy, C., and Caccamo, M. 2015. PolyMarker: A fast polyploid primer design pipeline. Bioinformatics 31:2038-2039.

Randhawa, M., Bansal, U., Valarik, M., Klocova, B., Dolezel, J., and Bariana, H. 2013. Molecular mapping of stripe rust resistance gene $\mathrm{Yr} 51$ in chromosome 4AL of wheat. Theor. Appl. Genet. 127:317-324.

Ray, D. K., Ramankutty, N., Mueller, N. D., West, P. C., and Foley, J. A. 2012 Recent patterns of crop yield growth and stagnation. Nat. Commun. 3: Article 1293.

Santra, D. K., Chen, X. M., Santra, M., Campbell, K. G., and Kidwell, K. K. 2008 Identification and mapping QTL for high-temperature adult-plant resistance to stripe rust in winter wheat (Triticum aestivum L.) cultivar "Stephens". Theor. Appl. Genet. 117:793-802.

Singh, A., Pandey, M. P., Singh, A. K., Knox, R. E., Ammar, K., Clarke, J. M., Clarke, F. R., Singh, R. P., Pozniak, C. J., DePauw, R. M., McCallum, B. D., Cuthbert, R. D., Randhawa, H. S., and Fetch, T. G. 2013. Identification and mapping of leaf, stem and stripe rust resistance quantitative trait loci and their interactions in durum wheat. Mol. Breed. 31:405-418.

Somers, D. J., Isaac, P., and Edwards, K. 2004. A high-density microsatellite consensus map for bread wheat (Triticum aestivum L.). Theor. Appl. Genet. 109:1105-1114.

Sourdille, P., Singh, S., Cadalen, T., Brown-Guedira, G. L., Gay, G., Qi, L., Gill, B. S., Dufour, P., Murigneux, A., and Bernard, M. 2004. Microsatellite-based deletion bin system for the establishment of genetic-physical map relationships in wheat (Triticum aestivum L.). Funct. Integr. Genomics 4:12-25.

Suenaga, K., Singh, R. P., Huerta-Espino, J., and William, H. M. 2003. Microsatellite markers for genes $\mathrm{Lr} 34 / \mathrm{Yr} 18$ and other quantitative trait loci for leaf rust and stripe rust resistance in bread wheat. Phytopathology 93: $881-890$.

Vazquez, M. D., Peterson, C. J., Riera-Lizarazu, O., Chen, X., Heesacker, A., Ammar, K., Crossa, J., and Mundt, C. C. 2012. Genetic analysis of adult plant, quantitative resistance to stripe rust in wheat cultivar "Stephens" in multi-environment trials. Theor. Appl. Genet. 124:1-11.

Vazquez, M. D., Zemetra, R., Peterson, C. J., Chen, X. M., Heesacker, A., and Mundt, C. C. 2015. Multi-location wheat stripe rust QTL analysis: Genetic background and epistatic interactions. Theor. Appl. Genet. 128:1307-1318.

Voorrips, R. E. 2002. MapChart: Software for the graphical presentation of linkage maps and QTL. J 615. J. Hered. 93:77-78.

Wang, H. 1996. Accumulation and distribution of dry matter on wheat variety Xinong1376. J. Northwest A\&F Univ. 5:31-34.

Wang, J. 2009. Inclusive composite interval mapping of quantitative trait genes. Acta Agron. Sin. 35:239-245.

Wang, S., Wong, D., Forrest, K., Allen, A., Chao, S., Huang, B. E., Maccaferri, M., Salvi, S., Milner, S. G., Cattivelli, L., Mastrangelo, A. M., Whan, A., Stephen, S., Barker, G., Wieseke, R., Plieske, J., Lillemo, M., Mather, D., Appels, R., Dolferus, R., Brown-Guedira, G., Korol, A., Akhunova, A. R., Feuillet, C., Salse, J., Morgante, M., Pozniak, C., Luo, M. C., Dvorak, J., Morell, M., Dubcovsky, J., Ganal, M., Tuberosa, R., Lawley, C., Mikoulitch, I., Cavanagh, C., Edwards, K. J., Hayden, M., and Akhunov, E. 2014. Characterization of polyploid wheat genomic diversity using a high-density 90000 single nucleotide polymorphism array. Plant Biotechnol. J. 12:787-796.

Weining, S., Ko, L., and Henry, R. J. 1994. Polymorphisms in the $\alpha$-amy1 gene of wild and cultivated barley revealed by the polymerase chain reaction. Theor. Appl. Genet. 89:509-513.

Wellings, C. R. 2011. Global status of stripe rust: A review of historical and current threats. Euphytica 179:129-141.

William, H. M., Singh, R. P., Huerta-Espino, J., Palacios, G., and Suenaga, K. 2006. Characterization of genetic loci conferring adult plant resistance to leaf rust and stripe rust in spring wheat. Genome 49:977-990.

Winfield, M. O., Allen, A. M., Burridge, A. J., Barker, G. L., Benbow, H. R., Wilkinson, P. A., Coghill, J., Waterfall, C., Davassi, A., Scopes, G., Pirani, 
A., Webster, T., Brew, F., Bloor, C., King, J., West, C., Griffiths, S., King, I., Bentley, A. R., and Edwards, K. J. 2016. High-density SNP genotyping array for hexaploid wheat and its secondary and tertiary gene pool. Plant Biotechnol. J. 14:1195-1206.

Wu, J., Huang, S., Zeng, Q., Liu, S., Wang, Q., Mu, J., Yu, S., Han, D., and Kang, Z. 2018a. Comparative genome-wide mapping versus extreme poolgenotyping and development of diagnostic SNP markers linked to QTL for adult plant resistance to stripe rust in common wheat. Theor. Appl. Genet. 131:1777-1792.

Wu, J., Liu, S., Wang, Q., Zeng, Q., Mu, J., Huang, S., Yu, S., Han, D., and Kang, Z. 2018b. Rapid identification of an adult plant stripe rust resistance gene in hexaploid wheat by high-throughput SNP array genotyping of pooled extremes. Theor. Appl. Genet. 131:43-58.

Wu, J., Wang, Q., Chen, X., Wang, M., Mu, J., Lv, X., Huang, L., Han, D., and Kang, Z. 2016. Stripe rust resistance in wheat breeding lines developed for central Shaanxi, an overwintering region for Puccinia striiformis f. sp. tritici in China. Can. J. Plant Pathol. 38:317-324.

Wu, J., Wang, Q., Kang, Z., Liu, S., Li, H., Mu, J., Dai, M., Han, D., Zeng, Q., and Chen, X. 2017. Development and validation of KASP-SNP markers for QTL underlying resistance to stripe rust in common wheat cultivar P10057. Plant Dis. 101:2079-2087.

Wu, J., Zeng, Q., Wang, Q., Liu, S., Yu, S., Mu, J., Huang, S., Sela, H., Distelfeld, A., Huang, L., Han, D., and Kang, Z. 2018c. SNP-based pool genotyping and haplotype analysis accelerate fine-mapping of the wheat genomic region containing stripe rust resistance gene Yr26. Theor. Appl. Genet. 131 1481-1496.

Zeng, Q., Wu, J., Huang, S., Yuan, F., Liu, S., Wang, Q., Mu, J., Yu, S., Chen, L., Han, D., and Kang, Z. 2019a. SNP-based linkage mapping for validation of adult plant stripe rust resistance QTL in common wheat cultivar Chakwal 86. Crop J. 7:176-186.

Zeng, Q., Wu, J., Liu, S., Chen, X., Yuan, F., Su, P., Wang, Q., Huang, S., Mu, J., Han, D., Kang, Z., and Chen, X. M. 2019b. Genome-wide mapping for stripe rust resistance loci in common wheat cultivar Qinnong 142. Plant Dis. 103 439-447.

Zeng, Q., Wu, J., Liu, S., Huang, S., Wang, Q., Mu, J., Yu, S., Han, D., and Kang, Z. 2019c. A major QTL co-localized on chromosome 6BL and its epistatic interaction for enhanced wheat stripe rust resistance. Theor. Appl. Genet. 132:1409-1424. 